\title{
Kernos
}

Revue internationale et pluridisciplinaire de religion grecque antique

$28 \mid 2015$

Varia

\section{A Different God? Dionysos and Ancient Polytheism}

\section{Francesco Massa}

\section{OpenEdition \\ Journals}

\section{Édition électronique}

URL : http://journals.openedition.org/kernos/2340

DOI : 10.4000/kernos.2340

ISSN : 2034-7871

\section{Éditeur}

Centre international d'étude de la religion grecque antique

\section{Édition imprimée}

Date de publication : 1 octobre 2015

Pagination : 273-277

ISBN : 978-2-87562-055-2

ISSN : 0776-3824

\section{Référence électronique}

Francesco Massa, «A Different God? Dionysos and Ancient Polytheism », Kernos [En ligne], 28 | 2015, mis en ligne le 01 octobre 2015, consulté le 23 septembre 2020. URL : http://journals.openedition.org/ kernos/2340 ; DOI : https://doi.org/10.4000/kernos.2340

Ce document a été généré automatiquement le 23 septembre 2020.

Kernos 


\title{
A Different God? Dionysos and Ancient Polytheism
}

\author{
Francesco Massa
}

\section{RÉFÉRENCE}

SCHLESIER Renate (éd.), A Different God? Dionysos and Ancient Polytheism, Berlin/Boston, De Gruyter, 2011. 1 vol. 17,5 × 24,5 cm, xiv+667 p., LXVIII pl. ISBN : 978-3-11-022234-0.

1 Entre 2011 et 2013, deux volumes issus de deux colloques qui ont eu lieu à Berlin et à Madrid ont centré leur attention sur le dieu Dionysos, en abordant plusieurs aspects relatifs à ses cultes et à ses mythes. Le volume madrilène édité par A. Bernabé, M. Herrero de Jáuregui, A.I. Jiménez San Cristóbal est axé sur la présence et le rôle de Dionysos chez les auteurs et dans les textes des mondes grec et romain ${ }^{1}$. En revanche, le volume berlinois édité par Renate Schlesier, dont il est question ici, choisit de mettre au cœur de la réflexion une question spécifique : «Dionysos est-il un dieu différent? ». L'historiographie des $\mathrm{XIX}^{\mathrm{e}}-\mathrm{x} \mathrm{x}^{\mathrm{e}}$ siècles a souvent fait de Dionysos un dieu 'à part' dans le polythéisme ancien, en soulignant son statut particulier, à la frontière entre norme et excès et en le dessinant comme la figure de l'altérité par excellence, incarnant l'Autre dans le monde divin des Grecs et des Romains. Dans le sillage des études des antiquisants, des philosophes et des anthropologues, le dieu du vin et de la folie est devenu, tour à tour, le filtre à travers lequel les intellectuels ont lu les grandes mutations culturelles et sociales du xxe siècle, de Mai 68 au Féminisme.

Comme le rappelle l'éditrice dans la Préface, le colloque a été organisé à l'occasion de l'exposition «Dionysos. Verwandlung und Ekstase», qui a eu lieu au Musée de Pergame, à Berlin, entre novembre 2008 et janvier 2010. La rencontre s'est attelée à trois questions fondamentales pour appréhender non seulement la nature de Dionysos, mais aussi les modes de fonctionnement des polythéismes antiques : Les Anciens ont-ils perçu Dionysos comme un dieu différent des autres divinités de leurs panthéons? Quelles sont les sphères d'action du dieu et dans quelle mesure ces sphères d'action le 
différencient-elles des autres dieux? Quel est l'impact des changements herméneutiques des savants modernes sur notre interprétation de cette divinité ? La dernière question ouvre donc la réflexion à la réception des imageries dionysiaques et aux divers courants historiographiques qui l'ont analysée.

Les 29 contributions sont organisées en six sections thématiques qui permettent d'une part de suivre les évolutions des cultes dionysiaques, de l'époque mycénienne à l'Antiquité tardive, et de l'autre, d'aborder les sujets les plus débattus concernant la figure de Dionysos. Non seulement le livre rassemble les meilleurs spécialistes des études dionysiaques, mais il présente une variété de méthodes d'analyse et d'approches d'une figure divine fondamentale des polythéismes anciens. Ce qui en ressort, c'est un panorama exhaustif des récits mythiques, des pratiques rituelles et des spéculations philosophiques autour de Dionysos. Le volume s'ouvre par l'étude de Glen W. Bowersock, hors section, consacrée d'une part à la représentation iconographique du premier bain de Dionysos, apparue au $\mathrm{I}^{\text {er }}$ siècle de notre ère, et d'autre part à des mises en parallèle de ces représentations iconographiques avec d'autres figures héroïques du monde romain, tels Achille et Alexandre le Grand. L'auteur réfléchit à la diffusion de ce schéma figuratif jusqu'à sa reprise dans les images de l'enfance de Jésus et l'interprète comme le signe du statut divin de ces personnages, une sorte de pratique rituelle qui marquerait le commencement de la vie divine.

La première section, Differences and Common Features, aborde de vastes questions concernant le culte de Dionysos et sa place dans le système complexe des relations polythéistes. Walter Burkert propose une réflexion globale sur le dieu Dionysos, en analysant des données qui vont de l'époque mycénienne jusqu'aux sources chrétiennes tardives. Henk S. Versnel pose des questions centrales sur les modes de fonctionnement du polythéisme grec et sur les relations entre les différentes formes cultuelles d'une même divinité : les épiclèses d'une divinité représentent-elles les diverses fonctions d'un seul dieu ou s'agit-il de dieux différents qui portent les mêmes noms? Versnel se positionne de manière critique à l'égard des travaux de Vernant et de ce qu'il appelle "son école " (p. 31) même si, pour ce faire, il se fonde presque exclusivement sur un article de Christiane Sourvinou-Inwood. Par ailleurs, l'auteur essaie de montrer que Dionysos se situe dans le même réseau divin que n'importe quel autre dieu ainsi que dans l'ambivalence entre l'un et le multiple. Susanne Gödde, en s'appuyant sur les travaux de J.-P. Vernant, développe l'idée d'une « différence mythologique " propre à Dionysos et reconnaît cinq éléments fondateurs de son statut particulier : la mère mortelle, la double naissance, la passion et la mort, la folie et les personnages qui se sont opposés au dieu (theomachoi). Albert Henrichs se concentre sur la question de l'épiphanie de Dionysos et montre comment l'image du dieu épiphanique est attestée notamment dans les sources littéraires, alors que son rôle dans les inscriptions est marginal, si on le compare à d'autres divinités comme Asclépios ou Isis. Des aspects plus spécifiquement cultuels sont pris en compte par deux autres études: Stella Georgoudi s'est penchée sur les sacrifices en l'honneur de Dionysos et, notamment, sur les sacrifices considérés comme "étranges " par une partie de la critique scientifique. Elle part de l'exemple du dossier concernant le culte de Dionysos Anthroporrhaistes, "Briseur d'homme ", attesté dans l'île de Ténédos et montre qu'il n'est pas possible de l'associer aux sacrifices humains ou à l'idée d'un démembrement mythique dionysiaque. L'étude de Georgoudi, tout en travaillant sur un cas d'étude, permet également de questionner un certain nombre d'idées reçues concernant la figure de Dionysos, comme par exemple le sacrifice dionysiaque appréhendé comme une forme 
de " cannibalisme », voire de "sacrement » ou la notion de « dieu souffrant » qui a été au centre des études des antiquisants jusqu'à la deuxième moitié du $\mathrm{xx}^{\mathrm{e}}$ siècle. Cet imaginaire avait contribué à la construction de la «différence» de Dionysos dans le cadre des polythéismes grecs. L'article de Marietta Horster analyse ensuite les aspects économiques liés aux pratiques dionysiaques afin de montrer que, du point de vue de l'organisation rituelle, les cultes bachiques ne présentent pas de différence significative par rapport aux autres divinités des mondes grec et romain.

Dans l'imaginaire des modernes, Dionysos est notamment le dieu du vin et la deuxième section du volume, Dionysiac Realms in Perspective, aborde plusieurs questions liées au vin et à sa consommation. Pauline Schmitt Pantel analyse la présence de Dionysos dans les banquets et son rapport avec les femmes : les textes et les images sont considérés au prisme des études de genre afin d'interpréter le rôle des scènes de mariages dans la production figurée du $\mathrm{vI}^{\mathrm{e}}$ siècle avant notre ère. Victoria Sabetai continue l'analyse de l'iconographie attique en proposant une étude de l'union entre Dionysos et Ariane sur les vases du peintre de Dinos et met en évidence l'émergence, dans la deuxième moitié $d u v^{e}$ siècle avant notre ère, d'une image renouvelée de l'amour et du mariage : son interprétation permet notamment de reconsidérer le dossier complexe de la fête des Anthestéries et d'abandonner l'idée d'un lien entre ces images et le moment de la hierogamia entre le dieu et la Basilinna, l'épouse de l'archonte roi athénien. Philippe Borgeaud focalise son attention sur l'épisode de la visite de Dionysos à Icarios et l'invention du vin qui s'ensuit : l'épiphanie dionysiaque en Attique ne s'accompagne pas de la résistance des opposants au culte de Dionysos et de leur punition. Borgeaud souligne qu'il est davantage question d'un récit autour d'un pròtos heuretès qu'il faut lire dans le contexte de la propagande athénienne du ve siècle. La section se referme sur l'article de Renate Schlesier qui aborde l'aspect sans doute le plus important du dieu Dionysos, la mania: le bakkheios theos est analysé d'abord dans les sources littéraires (Homère, Euripide, Platon), et ensuite suivant une perspective historiographique (Walter Otto, Eric R. Dodds) qui tout au long du xxe siècle a construit l'image du dieu autour de la centralité de la mania.

La troisième section, Specific Media of Transmission, propose une enquête sur les divers types de sources présentant l'image de Dionysos et sur leurs interprétations. Christian Wildberg ouvre la section par une analyse des spéculations philosophiques développées autour de la figure du dieu, en se fondant en premier lieu sur les fragments d'Héraclite puis en analysant l'impact de la réflexion de Platon et des philosophes platoniciens, notamment celui de Plotin. L'article est aussi l'occasion d'ouvrir le dossier du mythe de Dionysos et des Titans qui est connu, en grande partie, grâce aux relectures proposées par les philosophes de la période romaine. Même si la question n'est pas abordée directement, l'étude de Wildberg pose le problème du rapport entre " philosophie » et "religion», et de l'influence des discours philosophiques sur les conceptions religieuses des Anciens. Les études qui suivent se concentrent davantage sur les « supports » des témoignages. Il est d'abord question des représentations figurées avec l'article de Susanne Moraw qui analyse plusieurs thématiques bachiques dans l'art, comme les femmes, l'épiphanie, les batailles ou encore l'humanité du dieu. Thomas H. Carpenter continue l'enquête sur l'iconographie, mais il centre son attention sur la céramique apulienne et met en évidence les rapports du dieu avec le monde souterrain et la promesse d'une vie bienheureuse après la mort. Susan Guettel Cole se penche sur les données épigraphiques : l'étude est particulièrement importante dans le cadre de ce 
volume car elle dessine une image du dieu différente de celle donnée par les sources littéraires; la prise en compte des sources épigraphiques a souvent fait défaut aux études dionysiaques. Cole analyse plus particulièrement les pratiques de purification, ce qui permet de souligner l'intégration parfaite de Dionysos dans les contextes rituels grecs. De son côté, Dirk Obbink propose un panorama de la présence du dieu dans les papyrus, des poèmes d'Archiloque jusqu'au texte fragmentaire et complexe du Papyrus de Gurôb. Obbink analyse également un commentaire datant de la deuxième moitié du $\mathrm{III}^{\mathrm{e}}$ siècle avant notre ère qui contient des références au mythe de l'enfance de Dionysos.

7 Les deux sections qui suivent sont organisées sur la base d'une approche chronologique. La quatrième section, Theatre and the Polis of Athens, est consacrée à l'Athènes classique et notamment au phénomène dionysiaque par excellence, le théâtre. Natale Spineto se penche sur le rapport entre Dionysos et le théâtre, rapport qui anime le débat historiographique depuis l'Antiquité. Grâce à une approche socioanthropologique, Spineto propose une étude des groupes sociaux actifs dans les Grandes Dionysies athéniennes, en analysant trois aspects : la procession d'ouverture, le public des mises en scènes et le rôle des composantes de la société lors des festivités. Anton Bierl analyse un autre aspect de la question du rapport entre Dionysos et le théâtre, à savoir la présence et la fonction du dieu en tant que personnage dans les trois genres dramatiques: tragédies, comédies et drame satyrique. Il s'agit d'une vision d'ensemble très utile permettant de mieux éclairer le rôle dramaturgique du dieu du théâtre. Ensuite, Andrez L. Ford propose une étude détaillée du Dionysos « aux noms multiples » des Grenouilles d'Aristophane. La thématique du théâtre se clôt avec l'article très stimulant de Hans-Joachim Gehrke qui présente une réflexion intéressante sur l'opposition entre la vision structuraliste de Vernant et l'approche de Versnel: selon l'auteur, il faut interpréter le monde dionysiaque comme une coincidentia oppositorum entre la structure de la polis et une tentative de subversion de cette même polis.

Lorsque l'on passe à la cinquième section, Hellenistic and Roman Paradigms, le volume aborde les périodes hellénistique et romaine. Le premier article analyse le rôle politique de Dionysos dans la littérature latine, en abordant également la question complexe des rapports entre le Liber Pater romain et le Dionysos grec. Les exemples cités, issus notamment de l'époque augustéenne, renvoient en particulier à la divinisation des chefs politiques. Alexander Heinemann poursuit l'étude de la reprise et de la réutilisation romaine de la figure de Dionysos, mais il focalise son attention sur les représentations figurées et, plus particulièrement, sur la transformation des scènes de banquet. Anne-Françoise Jaccottet souligne à juste titre l'altérité dionysiaque dans les mythes anciens pour se demander comment l'altérité du dieu s'exprime dans le contexte urbain de la polis. Afin de répondre à ce problème capital qui touche autant aux pratiques religieuses qu'au système social, Jaccottet analyse des lieux de réunions des associations dionysiaques et, entre autres, les Iobakkhoi d'Athènes et les Boukoloi de Pergame. L'étude montre que l'altérité dionysiaque est toujours exprimée, mais de manière différente, car elle est parfaitement intégrée à la ville et insérée dans le système cultuel de la polis. Cornelia Isler-Kerényi travaille sur la représentation de Dionysos dans la ville de Pergame, ce qui permet d'étudier un exemple extraordinaire de transformation du dieu: celui-ci abandonne son rôle de divinité de la polis pour s'intégrer dans la structure bien plus large de l'Empire romain. Enfin, Christa Frateantonio ouvre une autre thématique fondamentale du monde impérial romain, en 
analysant ce qu'elle appelle le «syncrétisme » de Dionysos et Osiris chez Pausanias et plus généralement dans le monde gréco-romain.

9 La sixième et dernière section, Modern Reflexions, aborde les problèmes concernant la réception de Dionysos dans le monde contemporain. Michael Konaris se propose d'analyser la présence du dieu dans l'historiographie du $\mathrm{xx}^{\mathrm{e}}$ siècle, notamment dans trois domaines, les travaux sur la mythologie indoeuropéenne (W.Schwartz), l'approche historique (E. Curtius) et les études des anthropologues (A. Lang). D'après l'auteur, ces trois courants interprétatifs ont influencé l'interprétation de Dionysos aux époques suivantes. Oliver Leege analyse la façon dont l'image du Dionysos « dieu qui renaît » fut utilisée dans le cadre de la littérature grecque des $\mathrm{XIX}^{\mathrm{e}}-\mathrm{Xx}^{\mathrm{e}}$ siècles pour parler de la 'renaissance' de la Grèce en tant que nation indépendante. Roberto Sanchiño Martinez, de son côté, montre comment Dionysos a été utilisé comme modèle esthétique dans la philosophie $\mathrm{du} \mathrm{xx}^{\mathrm{e}}$ siècle ; il consacre en outre de très belles pages à la poésie de García Lorca. La section se termine avec l'étude de Froma I. Zeitlin qui propose d'abord un panorama des mises en scènes contemporaines des Bacchantes d'Euripide, en commençant par le célèbre Dionysos in 69 de Richard Schechner et offre ensuite une réflexion sur le Ion d'Euripide et sur le rôle important qu'y joue Dionysos.

La richesse documentaire et interprétative du livre est confortée par le nombre et la qualité des images contenues à la fin du volume (122 figures) et par l'acribie de trois index sur les noms, les sources anciennes et les termes, constituant ainsi un outil de travail fondamental pour les chercheurs travaillant sur le monde dionysiaque et, plus généralement, sur les religions des mondes antiques. Le volume contribue à remettre en cause un certain nombre de paradigmes herméneutiques utilisés par les savants des $\mathrm{XIX}^{\mathrm{e}}-\mathrm{XX}^{\mathrm{e}}$ siècles pour interpréter la figure de Dionysos. Grâce aux articles réunis par R. Schlesier, Dionysos retrouve sa place dans les dynamiques des polythéismes anciens. Certes, il reste la divinité fascinante que l'on connait par les récits mythiques et les relectures intellectuelles, mais les pratiques rituelles autour de cette figure divine s'intègrent parfaitement dans les systèmes cultuels civiques. Toutefois, même dans cette perspective générale, la diversité des approches contenues dans le présent volume confirme le polymorphisme de Dionysos et la possibilité d'interpréter sa nature et son statut de différentes manières, comme déjà Euripide nous l'avait bien montré dans la construction de l'intrigue de ses Bacchantes.

\section{NOTES}

1. A. Bernabé, M. herRero de JÁURegui, A.I. JimÉNEZ SAn cRistóbal (éd.), Redefining Dionysos, Berlin, 2013. 


\section{AUTEURS}

FRANCESCO MASSA

Université de Genève 